C 1996 IEEE. Personal use of this material is permitted. However, permission to reprint/republish this material

for advertising or promotional purposes or for creating new collective works for resale or redistribution to servers

or lists, or to reuse any copyrighted component of this work in other works must be obtained from the IEEE.

\title{
DAMPING RATE MEASUREMENTS IN THE SLC DAMPING RINGS*
}

\author{
C. Simopoulos, R.L. Holtzapple, \\ Stanford Linear Accelerator Center, Stanford University, Stanford, CA 94309 USA
}

\begin{abstract}
The transverse damping rates of the SLC electron and positron damping rings have been measured during the high current physics run at, $I=3.5 \times 10^{10}$ particles per bunch. The measurements, done over a period of two months, show large -uctuations exceeding the statistical uncertainty of each measurement. The longitudinal damping rate of the positron damping was also measured.
\end{abstract}

\section{DESCRIPTION OF THE OPTICS}

The synchrotron light produced in a bend magnet is re $\mathrm{rected}^{-}$ by a water cooled molybdenum mirror which resides inside the vacuum chamber. The light exits the vacuum chamber through a synthetic fused silica window. Subsequently it is collected by an achromatic lens of focal length $\mathrm{f}=1.33 \mathrm{~m}$ which functions as the objective lens of the optical system. The objective is located $1.47 \mathrm{~m}$ away from the emission point. The light is transported outside the ring vault by a succession of mirrors and through an evacuated pipe which is terminated on both ends by two synthetic fused silica windows. Finally the light reaches the fast gated camera lens [1], which was used in the transverse damping times measurements, and the image of the beam is formed on the photocathode. In order to increase the ®eld of view and prevent vigneting by the camera lens a large ®eld lens of $1.5 \mathrm{~m}$ focal length and $10 \mathrm{~cm}$ diameter was placed $1.5 \mathrm{~m}$ in front of the camera lens. The total distance between the objective and ®eld lens was $13.4 \mathrm{~m}$. The camera was focused on the ®eld lens which, within $1 \mathrm{~m}$, was also the position of the image of the beam formed by the objective. In the horizontal plane, the aperture was limited by a mask, $2 \mathrm{~cm}$ wide, placed in front of the objective. In the vertical direction the opening angle of synchrotron radiation determines the aperture. The focal length of the camera lens in the electron damping ring was chosen to be $300 \mathrm{~mm}$ and the whole system provided an overall magni ${ }^{\circledR}$ cation of $1: 2.5$. The positron damping ring camera lens had a focal length of $100 \mathrm{~mm}$ and the system provided a magni ${ }^{\circledR}$ cation of $1: 1$. The smaller magni®cation of the positron damping ring system was necessitated by the bigger size of the injected positron beam.

For the longitudinal damping time measurement a streak camera was used [2]. The optics con®guration for the streak camera differed because the small slit width required that the light was focused to a smaller spot. The $\mathrm{f}=1.5 \mathrm{~m}$ lens was removed and an $\mathrm{f}=50 \mathrm{~cm}$ lens was placed in front of the streak camera at a distance which provided the maximum illumination on the slit. The slit is imaged onto the photocathode of the streak camera by a pair of $\mathrm{f}=50 \mathrm{~mm}$ lenses which provide $1: 1$ magni ${ }^{\circledR}$ cation.

\footnotetext{
*Work supported by the Department of Energy, contract
}

The resolution of the system in the horizontal plane is $13 \mu \mathrm{m}$ determined by depth of ®eld [3]. The depth of @eld depends on the beam trajectory curvature and the horizontal aperture of the optical system, which is approximately $13.7 \mathrm{mrad}$. The diffraction limited resolution, using Raleigh's criterion, is $68 \mu \mathrm{m}$ determined by the natural opening angle of $4.0 \mathrm{mrad}$ at a wavelength of $550 \mathrm{~nm}$ [4]. The rms contribution due to diffraction is therefore estimated to be $34 \mu \mathrm{m}$. The critical wavelength for a $1.19 \mathrm{Gev}$ beam and bending radius $2.04 \mathrm{~m}$ is $0.68 \mathrm{~nm}$. The resolution in the vertical plane due to depth of @eld is determined by the length of the portion of orbit observed and the opening angle of synchrotron radiation in the vertical plane. The rms contribution is estimated to be $27 \mu \mathrm{m}$. The resolution due to the gated camera CCD pixel size is $13 \mu \mathrm{m}$ for the electron damping ring setup and $45 \mu \mathrm{m}$ for the positron damping ring. Adding all these contributions in quadrature the horizontal rms resolution is $18 \mu \mathrm{m}$ and $47 \mu \mathrm{m}$ for the electron and positron rings respectively. The rms contribution to the width in the vertical plane is $45 \mu \mathrm{m}$ and $64 \mu \mathrm{m}$.

\section{DATA ANALYSIS}

\section{A. Transverse}

The data were acquired in a random sequence of intervals of the time elapsed between injection and the gated camera trigger. This was done so that the data would not be biased by slow variations of the transverse beam size, during the data acquisition time, which was 20 min for 300 frames. Each frame of the beam image represents a different injected bunch. The electron bunch is stored for $8 \mathrm{msec}$ and the positron bunch is stored for $16 \mathrm{msec}$. The video signal of the gated camera was digitized by a transient waveform digitizer. The projections on the horizontal and vertical axes were @itted to a function of the form

$$
f\left(x ; A, B, C, \sigma, x_{0}\right)=\frac{A}{\sqrt{2 \pi} \sigma} e^{-\frac{1}{2}\left(\frac{x-x_{0}}{\sigma}\right)^{2}}+B+C x .
$$

The pedestal term was not constant because of the electrically noisy environment in the building where the camera was located. A $\chi^{2}$ minimization was performed for each frame with

$$
\chi^{2}=\sum_{i}^{n} \frac{\left(f\left(x_{i} ; A, B, C, \sigma, x_{0}\right)-v_{i}\right)^{2}}{v_{i}}
$$

Where $v_{i}$ is the rescaled digitized video signal so that the $\chi^{2}$ per degree of freedom distribution would peak around one. The statistical error on the measured sigma was determined from the correlation matrix derived from the minimization of the $\chi^{2}$. The square of the width was plotted against the time the bunch has spent circulating in the ring and the data was $\circledR^{\circledR}$ tted to a curve of the form

$$
\sigma(t)^{2}=\left(\sigma_{i n j}^{2}-\sigma_{e q}^{2}\right) e^{-\frac{2 t}{\tau}}+\sigma_{e q}^{2}
$$



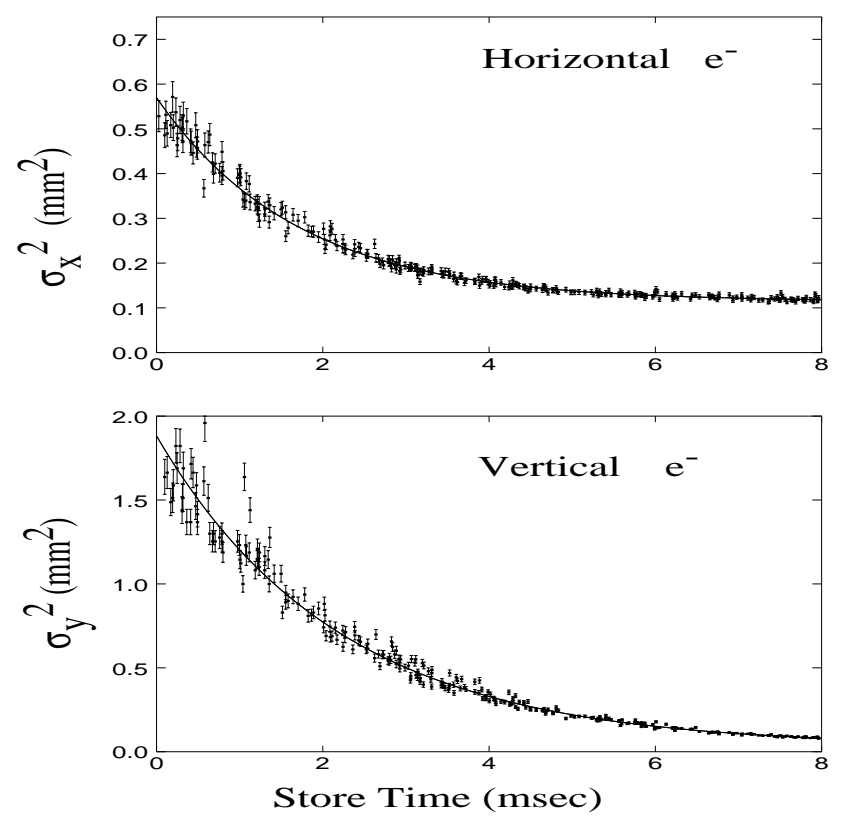

Figure 1. Sample of data for the electron damping ring. The vertical scale represents the size of the bunch image on the photocathode. The bunch itself is 2.5 times smaller

The ®tted parameters are $\sigma_{i n j}$ the width of the injected bunch, $\sigma_{e q}$ the equilibrium width of the bunch, and $\tau$ the damping time, see ${ }^{\circledR}$ gures 1,2 for a sample of data and $®$ ts.

\section{B. Longitudinal}

The analysis of the longitudinal damping time is similar. The projection of the image of the bunch on the time axis was $®$ tted to a function of the form

$$
\frac{A}{\sqrt{2 \pi} \sigma} e^{-\frac{1}{2}\left(\frac{x-x_{0}}{\sigma(1+5)}\right)^{2}}+B
$$

Where $S=|S| \frac{x-x_{0}}{\left|x-x_{0}\right|}$ representing the asymmetric shape of the bunch. The damping time is derived by a nonlinear exponential ®t. The data consist of ten measurements of bunch length ateach store time. The ten measurements at each point are averaged. The standard deviation of the ten measurements divided by $\sqrt{10}$ is used to weight the contribution of each point to the $\chi^{2}$ in the exponential ${ }^{\circledR}$ t, see ${ }^{\circledR}$ gure 3 .

\section{ERROR ANALYSIS}

\section{A. Transverse}

The uncertainty in the transverse measurements has statistical as well as contributions due to systematic errors. Despite the large contribution from resolution to the width measurements the damping time determination is not affected. The resolution adds in quadrature to the width, therefore the square of the width still follows an exponential damping law. However if the resolution depends on the transverse dimensions of the beam then the above statement is no longer true. In principle there is a dependence due to the fact that the depth of $®$ eld decreases as the horizontal dimension of the beam decreases but the variation is smaller
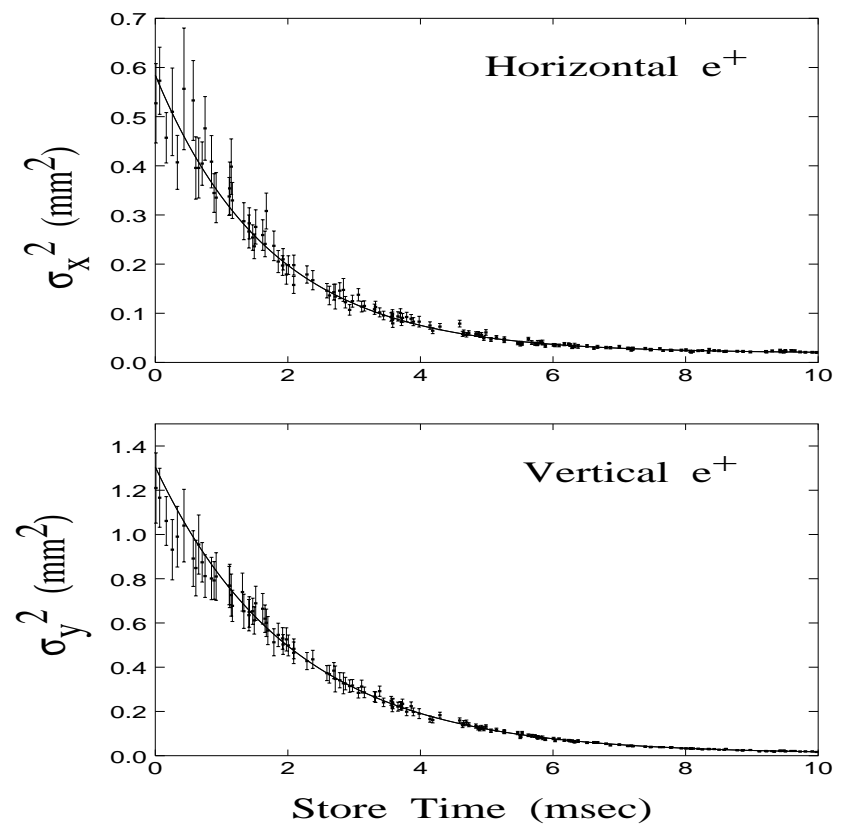

Figure 2. Sample of data for the positron damping ring. The vertical scale represents the real size of the bunch.

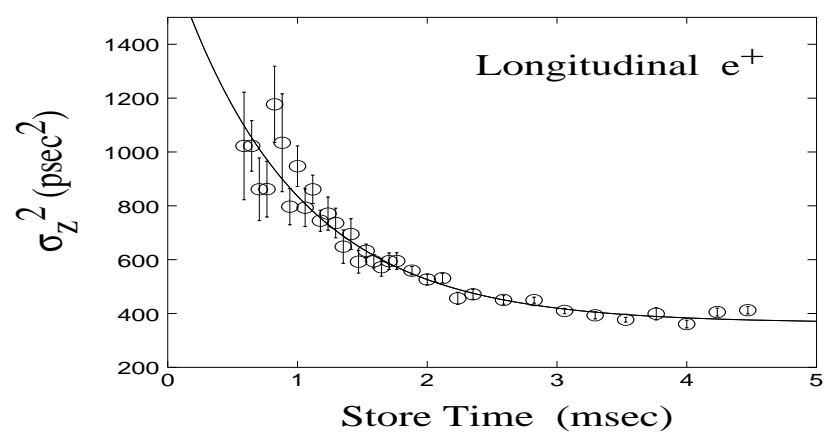

Figure 3. Longitudinal damping time data. The origin of the horizontal axis represents injection time.

than $1 \%$ due to the small angular acceptance of the optical system and the small angular spread of the synchrotron radiation in the vertical plane. Effects due to the damping of the angular divergence of the beam are also small since in the worst case of the injected positron bunch the angular divergence is $1.5 \mathrm{mrad}$, which is small compared to the light angular divergence.

The dynamic range for linear operation of the gated camera was rather limited. Even with the optimum amount of ®ltering, the photocathode showed signs of saturation as evidenced by the decreasing amount of light detected for increasing store time. The dependence of the width with intensity was measured and no clear plateau was found. Filtering was chosen so that the overall width variation due to this effect would stay below $10 \%$. From simulated data, this effect tends to decrease the measured damping times by $3 \%$.

The horizontal damping time can be in-uenced by the presence of dispersion which at the emission point is $\eta=5 \mathrm{~cm}$. The energy spread of the injected beam is $1 \times 10^{-2}$ which rapidly 

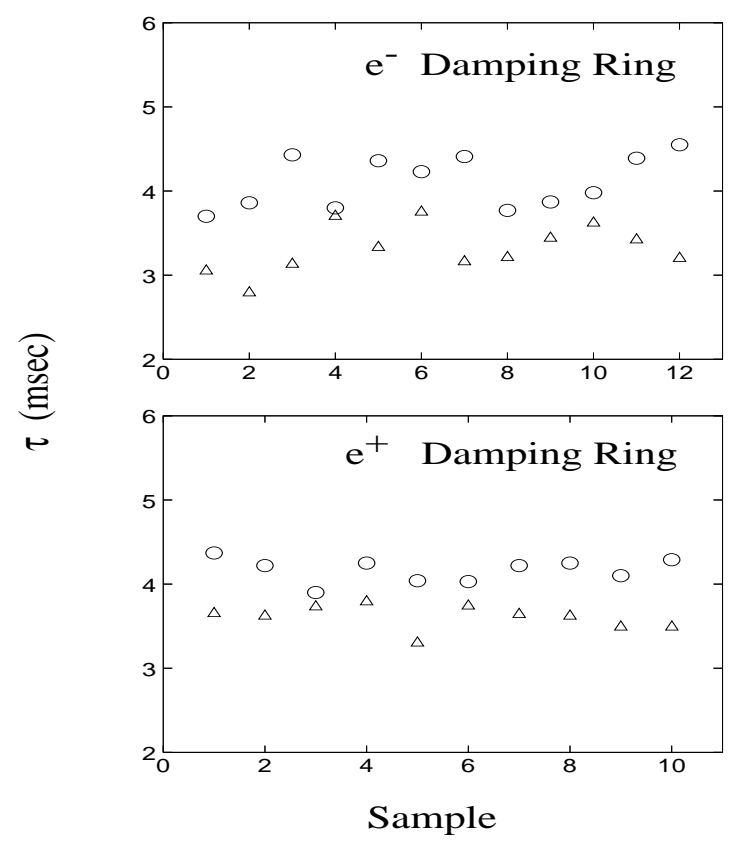

Figure 4. The horizontal damping times are represented by triangles and the vertical damping times by circles. The statistical error for each measurement is comparable to the size of the symbols

damps to the equilibrium energy spread $7 \times 10^{-4}$. In order to determine whether this effect is signi®cant, the data were ${ }^{\circledR} t$ ted to the sum of two exponentials where the damping time of one of the exponentials was ${ }^{\circledR} \mathrm{xed}$ to be equal to $1.8 \mathrm{msec}$, the longitudinal damping time. The ${ }^{\circledR}$ ts preferred a small negative weight for the second exponential. This indicates that any effect due to dispersion was masked by the nonlinear behavior of the gated camera and the low statistical weight of the points near injection.

In $®$ gure 4 the measurements are shown ordered in time. There is a large variation not explained by statistical errors indicating an additional unknown time dependent source of systematic error. Statistical errors are determined by the exponential $®$ ts, with properly weighted $\chi^{2}$, and on the average are $0.06 \mathrm{msec}$. For reasons of comparison with calculations of damping times [5] the measurements are averaged and summarized in the table above. The error quoted is the standard deviation of the corresponding set of measurements.

\section{B. Longitudinal}

Systematic errors due to photocathode saturation were eliminated by attenuating the light enough so that the camera was operated in the linear regime. The use of an interference $®$ lter centered at $500 \mathrm{~nm}$ and with a $40 \mathrm{~nm}$ bandwidth, ensured that the contribution to resolution from dispersion in the glass optics was negligible. There was only one measurement of the longitudinal damping time and the error quoted is the statistical uncertainty.

\section{CONCLUSIONS}

It is impossible on the basis of these measurements alone to distinguish whether the damping time -uctuations are due to instrumental effects or to orbit changes, or beam excitation from
Damping time measurements (msec)

\begin{tabular}{|l|c|c|c|}
\hline Ring & $\tau_{x}$ & $\tau_{y}$ & $\tau_{z}$ \\
\hline Electron & $3.32 \pm 0.28$ & $4.11 \pm 0.31$ & - \\
\hline Positron & $3.60 \pm 0.15$ & $4.17 \pm 0.14$ & $1.87 \pm 0.13$ \\
\hline Calculation & 3.52 & 3.56 & 1.79 \\
\hline
\end{tabular}

the extraction kicker magnets or interaction between the two bunches in the rings.

In order to understand the origin of the large -uctuations in the damping time measurements, and the discrepancy with the calculations, the orbit, tune and pressure in the vacuum chamber dependence should be studied.

The authors would like to thank F-J Decker, K. Jobe, P. Krejcik , M. Minty, M. Ross, R. Siemann, W. Spence and J. Spencer, for useful discussions and helpful suggestions and V. Brown for technical support during the course of this work.

\section{References}

[1] M. Minty et al., ${ }^{a}$ Using a fast-gated camera for the measurements of transverse beam distributions and damping times ${ }^{\circ}$, AIP Conference Proceedings No. 281, pp 158-167,1993.

[2] R.L. Holtzapple et al., ${ }^{a}$ Measurements of longitudinal dynamics in the SLC damping rings ${ }^{\circ}$, these proceedings.

[3] A.P. Sabersky, ${ }^{a}$ The geometry and optics of synchrotron radiation $^{\circ}$, Part . Acc., vol. 5, pp 199,1973.

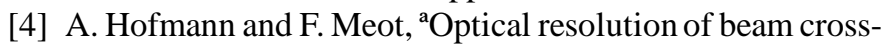
section measurements by means of synchrotron radiation ${ }^{\circ}$, Nucl. Instr. and Meth. vol. 203, pp 483-493, 1982.

[5] R. Early et al, aProposed emittance upgrade for the SLC damping rings ${ }^{\circ}$, SLAC-PUB-6559 